\title{
Dynamic Frame Sizing with Grouping Slotted Aloha for UHF RFID Networks
}

\author{
(DFSG)
}

\author{
Sobia Arshad ${ }^{1}$, Syed Muhammad Anwar, PhD. ${ }^{2}$, Mian Hammad Nazir ${ }^{3}$, Shumaila Khan ${ }^{4}$ \\ Department of Computer Engineering, University of Engineering and Technology, Taxila. Pakistan 1,2,4 \\ Comsats Institute of Information Technology (CIIT), Islamabad, Pakistan ${ }^{3}$
}

\begin{abstract}
Tag collision in a radio frequency identification system (RFID) is an important parameter that affects the overall system performance. Different algorithms have been proposed to efficiently utilize the slots during data transmission between reader and tags that need to be identified. Dynamic Frame slotted Aloha is a widely used anti collision algorithm that can be further divided into two main categories depending upon how the frame selection is performed. It has been found that dynamic frame sizing with grouping yields highly efficient results. In this study, the proposed algorithm modifies the mechanism used for dynamic grouping as implemented in the EPC global class 1 generation 2 algorithm and results have been presented showing improvement in the number of slots utilization and iterations required to successfully detect different number of tags.
\end{abstract}

\section{General Terms}

Algorithms, RFID Anti collision

\section{Keywords}

Tag anti collision, RFID, Slotted Aloha, EPC global class 1 generation 2, Dynamic Frame Sizing

\section{INTRODUCTION}

Radio frequency identification (RFID) system use radio waves to identify things without requiring line of sight communication as required for barcode systems. During the last decade RFID applications have been getting more and more attention due to its speed of identifying objects in large amounts [1]. RFID based systems have been used in a variety of applications a few examples are, asset tracking, library management, supply chain management, animal tracking, remote medical treatment, and counting of people in Hajj [2][3]. RFID system consists of two main components i.e. Interrogator and responder (tag). Tags are of three types i.e. passive, active and semi active tags. Passive tags are mostly employed due to ease of use being a battery less device [4].

The main goal in any RFID application is to identify a large number of objects in a very small time with high accuracy. When multiple products (each mounted with an RFID tag) come into the range of a single RFID reader, collision among tags occurs. This collision is known as tag to tag collision. Other types of collisions that could occur in an RFID system are reader to reader collision and reader to tag collision. To resolve this problem of collision among tags, many techniques have been proposed [5], most of which are Aloha based and Tree [5]. The major issues considered in a tag to tag anti collision protocol are accurate estimation of tags and optimization of frame size.

Two major categories of tag to tag anti collision protocols are, tree based protocols and Aloha based protocols. Tree based protocols are structured as a binary tree and offer $100 \%$ identification rate [6] The main disadvantage of tree based protocols is a large identification time [6]. When it comes to a large number of tags, the tree becomes very long, resulting in an increase in the identification time. A major category of Aloha based protocol is Framed Slotted Aloha (FSA) [5]. In FSA the reader sender sends the frame size to the tags, during an inventory round. The tags reply to the reader and get allotted a slot. Upon successfully transmitting the information embedded in the tag's memory, the slot is declared as successful. If the slot occupies more than one tag, it is declared as a collided slot. If the slot doesn't have any tag at all, that slot is declared as idle slot. Aloha based protocols have been found to operate at a system efficiency of $36.8 \%$ [7].

Framed slotted Aloha is further divided into two categories based on the adjustment of number of tags and frame size, known as Basic Framed Slotted Aloha (BFSA) and Dynamic Framed Slotted Aloha (DFSA) [8] - [9]. In BFSA, the frame size (number of slots) is fixed. During the inventory round when tags are to be identified, the frame size is transmitted to all tags. However the frame size doesn't change until all the tags inventoried. Due to its fixed frame size throughout the process of inventory, it is called as Static framed slotted aloha [9]. In DFSA, the frame size is selected in real time that is updated by estimating the number of tags and with the help of probabilistic methods. [9]. the frame size is selected so as to keep the system efficiency optimal.

Organization of paper as follows: Section 2, 3 contains literature review related to DFSA and EPC class1 gen2 Standard. Section 4 and 5 describes details of proposed method with performance analysis. We conclude the paper in section 6 .

\section{OVERVIEW OF DIFFERENT DFSA TECHNIQUES}

DFSA is used mostly in RFID systems to decide optimal frame size. It adjusts frame size on the basis of idle ratio and collision ratio because it doesn't have information about number of tags. The schemes that estimate number of tags on the basis of collision and idle ratio and then adjust the frame size accordingly; are discussed below. 


\subsection{Frame Sizing Techniques}

Schoute [10] proposed DFSA algorithm based on Poisson distribution. To estimate the number of unidentified tags, he multiplied the number of collision slots with the expected value of 2.39 per collision slot.

$$
B_{t}=2.39 c
$$

In Equation 1 ' $c$ ' is the number of collision slots and $\mathrm{Bt}$ is estimated number of tags.. The next frame size $N_{n x t}$ was calculated using,

$$
N_{n x t}=S_{\text {succ }}+B_{t}
$$

Number of successfully transmitted tags is $S_{\text {succ }}$. This method gives worst results when difference between number of tags and slots is big. Lower Bound backlog estimation technique [10] states that estimation function is obtained with the assumption that whenever a collision occurs, it involves at least two tags,

$B t=2 c$

This technique only performs well when number of tags and slots are close. Vogt [11]-[12] proposed estimating number of tags by using Lower bound backlog estimation.

$B t=C 1+2 C \geq 2$

C1 is the number of successful slots. This estimation fails in sever collision conditions. Vogt considered Markov's Model for frame size estimation. Frame size is decided on the basis of low and high values of ' $n$ total number of tags]. Moreover it employs minimum distance to choose an optimal frame size. An optimal intervals table for frame size was presented as:

Table1. Tag Grouping Up to 10 Iterations

\begin{tabular}{|l|l|l|l|l|l|l|l|l|}
\hline Slots (N) & $\mathbf{1}$ & $\mathbf{4}$ & $\mathbf{8}$ & $\mathbf{1 6}$ & $\mathbf{3 2}$ & $\mathbf{6 4}$ & $\mathbf{1 2 8}$ & $\mathbf{2 5 6}$ \\
\hline Low & - & - & - & 1 & 10 & 17 & 51 & 112 \\
\hline High & - & - & - & 9 & 27 & 56 & 129 & $\infty$ \\
\hline
\end{tabular}

It is complex and hard to find the best minimum value. Zhai \& Wang [13] presented a probabilistic method based on Binomial distribution given as,

$$
\begin{aligned}
& B_{n, N}(r)=\left(\begin{array}{l}
n \\
r
\end{array}\right) \cdot(1 / N)^{r} \cdot(1-1 / N)^{n-r} \\
& B_{n, N}(r)=N \cdot\left(\begin{array}{l}
n \\
r
\end{array}\right) \cdot(1 / N)^{r} \cdot(1-1 / N)^{n-r}
\end{aligned}
$$

Equation (6) is for ' $\mathrm{N}$ ' is the total number of slots for ' $\mathrm{r}$ ' tags. Here ' $n$ ' is the total number of tags. Chen \& Lin [14] used binomial distribution to identify number of tags. They also used collision slots, idle slots and successful slots to estimate frame size.

$N=S+E+C$

$$
B_{t}=(N-1) \cdot(a 1 \div a 0)
$$

S, E, C is for successful slots, Empty slots and collision slots respectively. $\mathrm{N}$ is the Total number of slots. ' $a 1$ 'and ' $a 0$ ' are for successful and empty slots respectively.

\subsection{Frame sizing with tag grouping techniques}

System efficiency degrades when there are large numbers of tags due to complex adjustment of frame size[15]. Frame size has limitations, so when there is infinite number of tags, adjustment of tags with the frame size is necessary to maintain maximum system efficiency. This is done by grouping of tags. Tag grouping is done by two main methods, static and dynamic tag grouping. In static tag grouping schemes there are large number of tags that are divided into same size of groups [16]. In Enhanced Dynamic Frame Slotted Aloha (EDFSA) [16] tags are divided into a number of groups that can be identified on a group by group basis. It takes modulo of unread tags to frame size and tags are divided into groups based on this operation. EDFSA degrades efficiency because it never adjusts frame size until all tags in one group falls below a specific threshold .In comparison, dynamic tag grouping schemes, tags of a particular frame (group) are identified and grouping is performed again on remaining tags and then identified [17].

\section{EPC GLOBAL CLASS 1 GENERATION 2 STANDARD}

EPC (Electronic Product Code) global class 1 generation 2 ultra high frequency (UHF) RFID protocols [4] specifies physical and logical interactions between tags and readers. It also defines the commands and operating methods between tags and readers and present an anti collision algorithm for multi tag environment. This standard offers faster, highly reliable, more robust and enhanced secured results as compared to generation 1 protocol [4]. It uses slotted aloha based on Q algorithm. In RFID systems operating at $860 \mathrm{MHz}-$ $960 \mathrm{MHz}$ frequency, reader transmits information signal (Query Command) of 22 bit to tag. Tag receives information and signal energy to energize itself, as passive tags don't have their own batteries or power source.

Query command has a field 'Q' for frame size selection. Tag selects a value from $0-2^{Q-1}$. The tag which selects 0 , replies with a 16 bit identification, ID, and RN16 command. All other tags decrement their slot counters by one and wait for their turn. If a tag successfully transmits 16 bit ID without collision, then reader sends an 18 bit ACK command back to that tag. After receiving ACK, that tag transmits data including EPC of 96-256 bits and 16 bit cyclic redundancy check (CRC).Reader send a command of 4-bit (Query Rep) to all tags to decrement their slot counter by one. Any tag reaching 0 , starts communication with the reader. The flow diagram is depicted in Figure 1.

EPC global class 1 generation 2 standard is very simple to implement and a compact representation of frame size $=2^{Q}$. The drawback is the fact that, frame size cannot be adjusted 
linearly either in case of extreme collision or extreme idle slots. It results in system efficiency degradation and identification time enhancement.

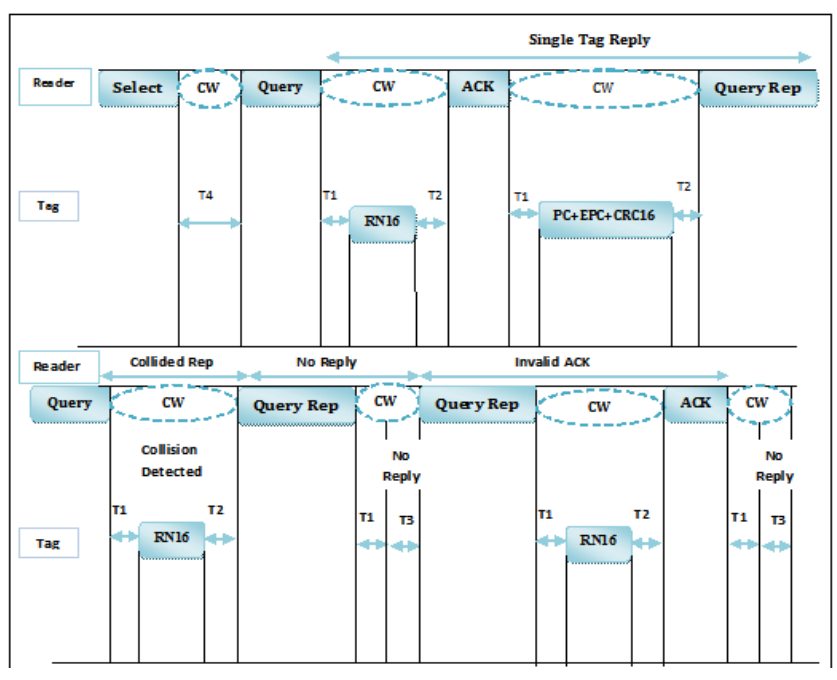

Fig 1: EPC global class 1 generation 2 Standard

\section{Proposed Dynamic Frame Slotted Aloha}

\subsection{Preliminary concepts \& Mathematical}

\section{analysis}

Binomial distribution provides the probability of $j$ tags in one slot, given as,

$$
P(X=j)=\left(\begin{array}{c}
n \\
j
\end{array}\right)\left(\frac{1}{N}\right)^{j}\left(1-\frac{1}{N}\right)^{n-j}
$$

In Equation (9), $N$ is the number of slots and $\mathrm{n}$ is the number of tags. The probability when no tag exists can be found by setting $j=0$,

$$
\mathrm{P}(\mathrm{X}=0)=\left(1-\frac{1}{\mathrm{~N}}\right)^{\mathrm{n}}
$$

The probability of only one tag in one slot is given by setting $j=1$,

$$
P(X=1)=n \cdot\left(\frac{1}{N}\right) \cdot\left(1-\frac{1}{N}\right)^{n-1}
$$

The probability that more than one tag exists in a single slot $(j>1)$ is,

$$
P(X>1)=1-P(X=0)-P(X=1)
$$

Equations 10-12 represent the probability of idle slot, successful allocation and collision respectively. Multiplying these equations by $\mathrm{N}$ give their expected values as,

$$
\begin{aligned}
& P_{0}^{N, n}=N \cdot P(X=0) \\
& P_{1}^{N, n}=N \cdot P(X=1) \\
& P_{>1}^{N, n}=N \cdot P(X>1)
\end{aligned}
$$

System efficiency is the ratio of the number of slots with one tag to the current frame size,

$$
\mu=\text { System Efficiency }=\frac{P_{1}^{N, n}}{N}=\mathrm{n} \cdot\left(\frac{1}{\mathrm{~N}^{2}}\right) \cdot\left(1-\frac{1}{\mathrm{~N}}\right)^{\mathrm{n}-1}
$$

To find Maximum efficiency, let $\frac{d \mu}{d N}=0$; that is

$$
\begin{gathered}
\frac{d \mu}{d N}=(n-1) \cdot\left(\frac{n}{N^{2}}\right)\left(1-\frac{1}{N}\right)^{n-2}-\left(\frac{n}{N^{3}}\right)\left(1-\frac{1}{N}\right)^{n-1}=0 \\
N \cong n \\
\mu_{\max }=\left\{\begin{array}{cl}
1 & , n=1 \\
\left(1-\frac{1}{N}\right)^{N-1}, & n>1
\end{array}\right.
\end{gathered}
$$

If $n \gg>1$, we have the condition for maximum efficiency, the maximum system efficiency is listed in Table 2, for different values of ' $n$ ' and given as,

Table 2. Maximum System Efficiency

\begin{tabular}{|c|c|c|c|c|c|c|}
\hline $\mathbf{n}$ & 1 & 2 & 4 & 8 & 16 & 32 \\
\hline $\boldsymbol{\mu}_{\max }$ & 1 & 0.5 & 0.42 & 0.393 & 0.38 & 0.374 \\
\hline $\mathbf{n}$ & 64 & 128 & 256 & 512 & 1024 & 2048 \\
\hline $\boldsymbol{\mu}_{\max }$ & 0.371 & 0.369 & 0.368 & 0.368 & 0.368 & 0.371 \\
\hline
\end{tabular}

According to EPC global class 1 generation 2 standards, the interrogator sends Query command to all tags. Tags that are to be identified, should participate in the inventory round. It is assumed that tags are perfectly estimated. In the Query command there is field, ' $\mathrm{q}$ ' for frame size selection that could range from $0-2^{\mathrm{q}-1}$. Proposed Algorithm for dynamic grouping is presented in Figure 2 and in Table 3.

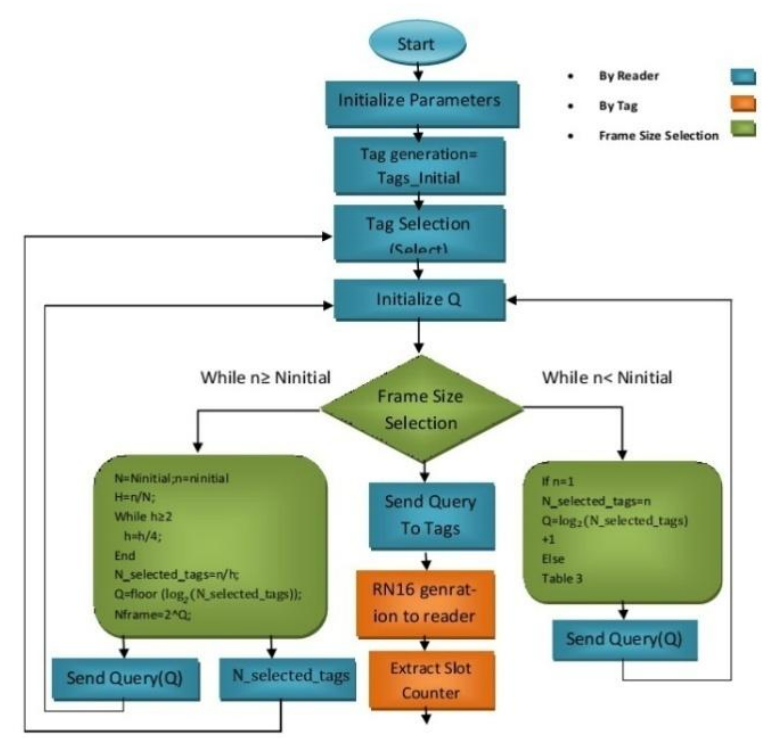

Fig 2: Flow chart of Proposed Scheme

Table 3. Frame size for less than Ninitial

\begin{tabular}{|c|c|c|}
\hline $\mathbf{n}$ & $\mathbf{Q}$ & Nframe \\
\hline $\mathbf{2 - 5}$ & 2 & 4 \\
\hline $\mathbf{6 - 1 1}$ & 3 & 8 \\
\hline $\mathbf{1 2 - 2 2}$ & 4 & 16 \\
\hline $\mathbf{2 3 - 4 4}$ & 5 & 32 \\
\hline $\mathbf{4 5 - 8 8}$ & 6 & 64 \\
\hline $\mathbf{8 9 - 1 7 6}$ & 7 & 128 \\
\hline $\mathbf{1 7 7 - 2 5 5}$ & 8 & 256 \\
\hline
\end{tabular}


Table 4 shows the decrease in iterations and result of tag grouping. Ninitial is set equal to 256 and N_selected_tags are selected using SELECT command. Q is set in QUEY command. Tag that rolls 0 replies immediately the 16 bit ID, RN16. Number of tags that would participate in the next round is calculated using,

Tags $_{\text {initial }}=$ Tags $_{\text {remaining }}=$ Tags $_{\text {initial }}-$ Tags $_{\text {succ }}$

In this Tags succ $_{\text {and }}$ Tags $s_{\text {idle }}$ can be calculated by multiplying Equation (11) and (10) by N_selected_tags respectively.

$$
\begin{aligned}
& \text { Tags }_{\text {succ }}=\mathrm{N}_{\text {selected }_{\text {tags }}} * P(X=1) \\
& \text { Tags }_{\text {idle }}=\mathrm{N}_{\text {selected }_{\text {tags }}} * P(X=0)
\end{aligned}
$$

\section{Table4. Tag Grouping Up to 10 Iterations}

\begin{tabular}{|c|c|c|c|c|c|c|c|c|c|c|}
\hline Tags & G1 & G2 & G3 & G4 & G5 & G6 & G7 & G8 & G9 & G10 \\
\hline $\mathbf{1 0 0}$ & 53 & 29 & 17 & 11 & 8 & 5 & 3 & 1 & & \\
\hline $\mathbf{2 0 0}$ & 106 & 59 & 35 & 23 & 11 & 8 & 5 & 3 & 1 & \\
\hline $\mathbf{3 0 0}$ & 206 & 112 & 65 & 41 & 29 & 17 & 11 & 8 & 5 & 3 \\
\hline $\mathbf{4 0 0}$ & 306 & 212 & 118 & 71 & 47 & 23 & 11 & 8 & 5 & 3 \\
\hline $\mathbf{5 0 0}$ & 406 & 312 & 218 & 124 & 77 & 53 & 29 & 17 & 11 & 8 \\
\hline $\mathbf{1 0 0 0}$ & 623 & 246 & 152 & 105 & 58 & 34 & 22 & 16 & 10 & 7 \\
\hline $\mathbf{1 5 0 0}$ & 1123 & 746 & 369 & 275 & 181 & 87 & 63 & 39 & 27 & 15 \\
\hline $\mathbf{2 0 0 0}$ & 1623 & 1246 & 869 & 492 & 398 & 304 & 210 & 116 & 69 & 45 \\
\hline $\mathbf{2 5 0 0}$ & 993 & 616 & 239 & 145 & 98 & 51 & 27 & 15 & 9 & 6 \\
\hline
\end{tabular}

\section{SIMULATION RESULTS \& PERFORMANCE ANALYSIS}

This section shows simulation Results. All results obtained from MatLab. Table 4 shows the dynamic grouping with frame sizing. All results were obtained by keeping initial frame size of 256 (Ninitial=256).

We compared our results with BFSA and EDFSA. We chose BFSA because it works on the basis of Static Frame Sizing. We implemented EDFSA because it works on the basis of dynamic frame sizing with tag grouping. Implementing BFSA, frame size was 256 until all tags succeeded. Implementing EDFSA, frame size varies from 8 to 256. There was no relation to calculate remaining number of tags for next iteration in EDFSA. We implemented the Eq (17) and (18) for this purpose.

\subsection{Identification Time}

We associate identification/Execution time by the number of iterations and by no. of slots. We compared BFSA and EDFSA with our proposed Algorithm. Figure 3 shows the resulting no. of slots utilized up to 2500 tags and Figure 4 shows the results for number of iterations.

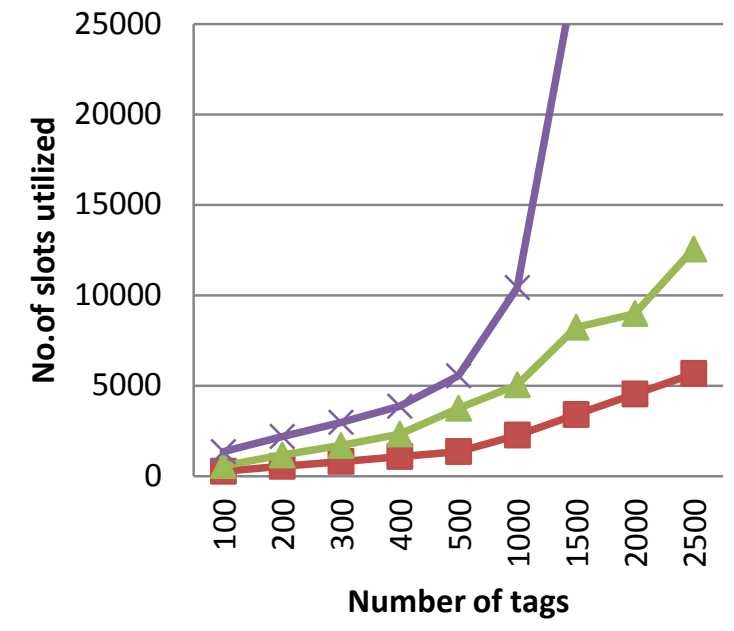

Propsed Scheme $\longleftarrow$ EDFSA $\longleftarrow$ BFSA

Fig 3: Number of Tags vs. Number of Slots for EDFSA, BFSA and Proposed Scheme

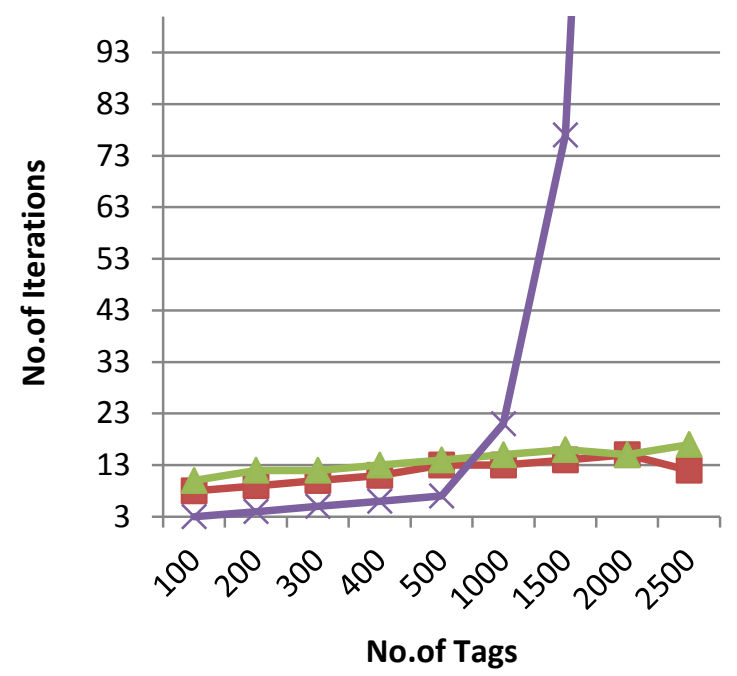

Propsed Scheme $\multimap$ EDFSA $\asymp$ BFSA

Fig 4: Number of Tags vs. Number of Iterations for BFSA, EDFSA and Proposed Scheme

\subsection{Collision Ratio}

To determine Collision Ratio, we presented a new relation as

$$
\text { Collision Ratio }=\frac{\text { Ntotal }- \text { Tags }_{\text {succ }_{\text {to }}}-\text { Tags }_{\text {idle }}}{\text { Ntotal }}
$$


Figure 5 shows the collision ratio for various numbers of tags. Its constant value is 0.2642 .Our Proposed Algo shows optimal results for this collision ratio also.

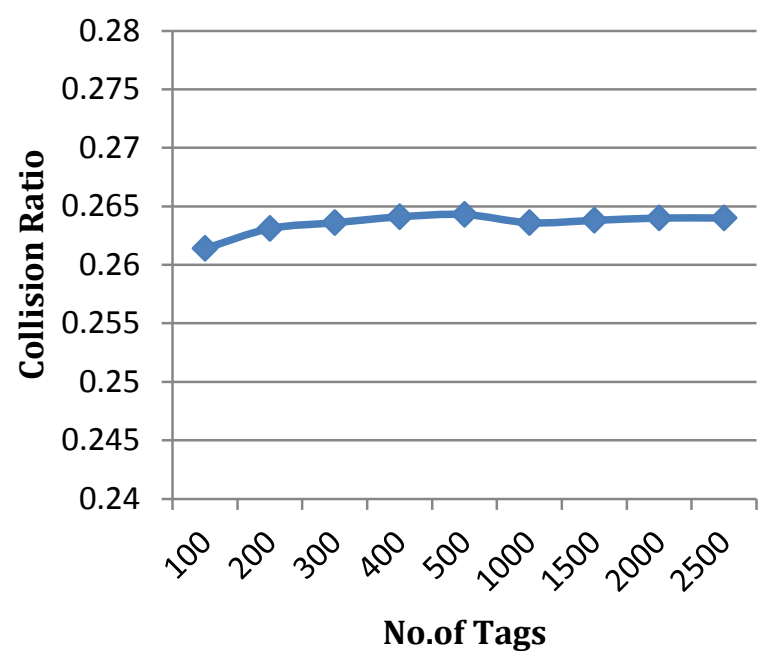

$\sim$ Propsed Scheme

Fig 5: Number of Tags vs. Collision Ratio

\subsection{System Efficiency}

System Efficiency can be calculated by using the relation

System Efficiency $=\frac{\text { No.of Successful Slots }}{\text { Total No.of slots }}=\frac{\text { Tags }_{\text {succ }}}{\text { Ntotal }}$

Figure 6 shows the system Efficiency for BFSA, EDFSA and for our Proposed Method. System Efficiency varies in the start from 0.377 to 0.371 , towards end it varies from 0.369 to 0.368 . These values are very close to 0.368 for large number of tags. When large number of tags to be identified, system efficiency will be about 0.368 .

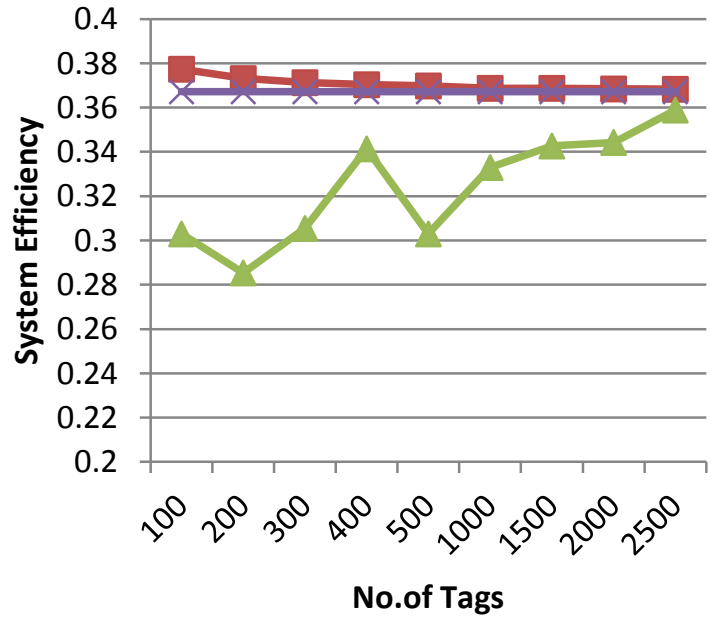

Propsed Scheme $\leftarrow$ EDFSA $\leftarrow$ BFSA

Fig 5: Number of Tags vs. System Efficiency for BFSA, EDFSA and Proposed Scheme

\section{CONCLUSION}

A new Dynamic frame sizing with Tag grouping algorithm is proposed. We presented a clear relation to read unidentified number of tags. In EDFSA there was no relation to obtain number of unread tags. A new modified relation for collision ratio is presented. We associated identification time with number of iterations and number of slots utilized. All graphs are clearly presented in comparison with BFSA and EDFSA. Collision ratio and system efficiency results are very close optimal values. Our Proposed Algorithm can be implemented with very little change in protocol.

\section{ACKNOWLEDGEMENT}

We acknowledge and thank Engr. Hammad Nazir for their full support for this research. We also thank University of Engineering \& Technology Taxila for providing such a remarkable research environment for Full time MSc. Students.

\section{REFERENCES}

[1] R.Want, “An Introduction to RFID Technology," Proc. Published by the IEEE CS and IEEE ComSoc, January March 2006.

[2] Applications of RFID. Texas Instruments. [Online] http://www.ti.com/rfid/applications.shtml.

[3] M. Naser, M. Rafi, and R. Budiarto, "Security Considerations In Embedding RFID in Hajj System," European Journal of Scientific Research, vol. 42, no. 1, pp. 133-138, 2010

[4] EPC. radio-frequency identity protocols class-1 generation- 2 UHF rfid protocol for communications at $860 \mathrm{MHz}-960 \mathrm{MHz}$ version technology.Version1.2.0. s.l. EPCglobal, 2008. httpllwww.epcglobalinc.orglstandards.

[5] S. Maharjan, "RFID and IOT: An overview," Simula Research Laboratory,University of Oslo, Sep.2010.

[6] C.Yi-Wang, and C.Chung-Lee, "A FGrouping-Based Dynamic Framed Slotted ALOHA Anti-Collision Method with Fine Groups in RFID Systems," in the Proc. of IEEE International Conference on Future Information Technology (FutureTech), 2010.

[7] D. K.-Klair, K.Wu-Chin, and R.Raad, "A Survey and Tutorial of RFID Anti-Collision Protocols," IEEE Communications Surveys \& Tutorials, vol. 12, no.3, pp. 2010.

[8] H.Tsao, D.Deng, H.Wen-Wang and J.Chang, "Runtime Optimization of Framed Slotted ALOHA Based RFID Systems," 6th International Symposium on Wireless and Pervasive Computing (ISWPC), pp. 2011.

[9] G.Shu-qin, W.Wu-chen, H.Li-gang and Z.Wang, "Anticollision Algorithms for Multi-Tag RFID," [book auth.] Cristina Turcu. Radio Frequency Identification Fundamentals and Applications, Bringing Research to Practice . Beijing 100022,: VLSI and System Lab, Beijing University of Technology, , 2010.

[10] P.Pupunwiwat, and B.Stantic , "Dynamic Framed-Slot ALOHA Anti-Collision using Precise Tag Estimation Scheme," in the Proc. of Twenty-First Australasian Conference on Database Technologies, vol. 104, pp. 19$28,2010$. 
[11] H.vogt, "Efficient object identification with passive RFID tags," in the Proc.of International conference on pervasive computing, LNCS Springer -Verlag, 2002.

[12] H.vogt, "Multiple object identification with passive RFID tags," in the Proc. of IEEE International Conference on Systems, Man and Cybernetics, vol. 3, 2002.

[13] J.Zhai, and G.Wang, "An Anti-collision Algorithm Using Two-Functioned Estimation," Computational Science and Its Applications, 2005.

[14] W.chen, and G.H.Lin, "An efficient anti-collision method for tag identification in RFID", IEICE TRANS.COMMUNICATIONS, Vol. 89,2006.
[15] J.Eom, and T.Lee,"An Efficient Framed-Slotted ALOHA Algorithm with Pilot Frame and Binary Selection for Anti-Collision of RFID Tags". IEEE communications letters, Vol. 12, 2008.

[16] S. Lee, S. Joo, C. Lee, "An Enhanced Dynamic Framed Slotted ALOHA Algorithm for RFID Tag Identification," in the Proc. of International Conference on Mobile and Ubiquitous Systems: Networking and Services (MOBIQUITOUS), pp. 166-174, 2005.

[17] X Huang, "An Improved ALOHA Algorithm for RFID Tag Identification", Knowledge-Based Intelligent Information and Engineering Systems [Book] Berlin Heidelberg, Springer-Verlag, vol. 4253, pp. 1157-1162, 2006. 